
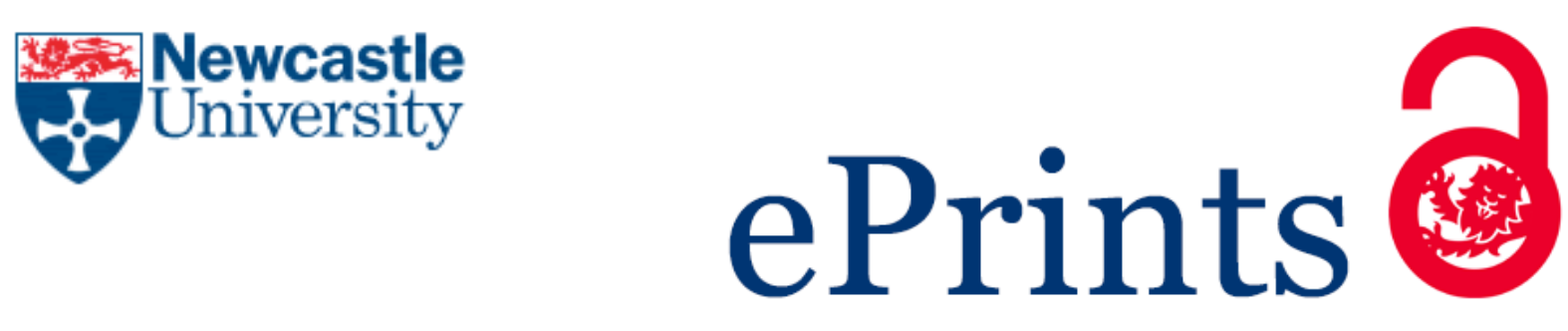

Rundle SD, Robinson D, Strang D, Wienroth M.

ATRIA: A sound installation exploring the interface between art, science and technology by remapping cardiovascular development.

Leonardo 2014, 47(5), 443-449.

\title{
Copyright:
}

(C)2014 ISAST.

DOI link to article:

https://doi.org/10.1162/LEON a 00868

Journal homepage:

http://www.mitpressjournals.org/loi/leon

Date deposited:

$06 / 06 / 2017$ 


\section{ATRIA: A Sound Installation Exploring the Interface between Art, Science and Technology by Remapping Cardiovascular Development}

\author{
Simon D. Rundle, \\ Deborah Robinson, \\ David Strang and \\ Matthias Wienroth
}

A B S T R A C T

AtRIA was an immersive sound installation that was the result of a dynamic, reflective dialogue between artist Deborah Robinson and biologist Simon Rundle during Robinson's residency within the Marine Biology and Ecology Research Centre, Plymouth University. The work drew on theoretical ideas in developmental biology and the sociology of science and practical laboratory investigations in developmental physiology. Data from videos of snail embryos used to map physiological function during development using conventional (scientific) diagrams were "remapped" as sound projections into a threedimensional building space, transposing scientific knowledge into a public experience as a "mutable mobile." desire of Deborah Robinson and Simon Rundle to produce an artwork that emerged from a reflective dialogue between an artist and a scientist. The aim was to develop artwork that would articulate an aspect of the scientific work undertaken at the Marine Biology and Ecology Research Centre (MBERC) at Plymouth University. Robinson's appointment as artist in residence in the MBERC allowed her continuous access to a scientific research environment for a year. Regular (biweekly) meetings were held during this time, which allowed Robinson to learn about and follow research into developmental biology being carried out in the laboratory. The exchange of ideas was enhanced by the fact that most of these meetings were held within the research center, allowing ready access to the laboratory environment, the animals being studied and scientific literature. Robinson also sat in on meetings between researchers. At the same time, Rundle and members of his research team attended meetings in the art studio and were involved in discussions about practical aspects of the development of the artwork, including technical discussions with the sound artist David Strang.

At the start of her residency, Robinson had no preconceived ideas of what form her art would take. The relatively long duration (12 months) of the residency gave sufficient time for her work to evolve through a dynamic exchange of ideas that ran in parallel with the scientific progress within the labora-

Simon Rundle (biologist), Marine Biology and Ecology Research Centre, School of Marine Science and Engineering, Plymouth University, PL4 8AA, U.K Email: <srundle@plymouth.ac.uk>.

Deborah Robinson (artist), Centre for Media, Art \& Design Research, School of Art and Media, Plymouth University, PL4 8AA, U.K.

David Strang (artist), Centre for Media, Art \& Design Research, School of Art and Media, Plymouth University, PL4 8AA, U.K.

Matthias Wienroth (sociologist), Northumbria University Centre for Forensic Science, Northumbria University, NE1 8ST, U.K.

See $<$ www.mitpressjournals.org/toc/leon/47/5> for supplemental files associated with this issue. tory. She was able to track how the research progressed: how data were generated, displayed and discussed; how new ideas arose; how the scientific questions being addressed became redirected. Discussions also drew heavily on the theory behind both the science and the art. Subsequent reflective periods then allowed ideas to be melded and the collaborative science-art project to take new directions. In the following account, the scientific and philosophical underpinnings to the ATRIA project are outlined and then the practice behind the artwork is described. The interaction of science, technology, art and social practices are considered from a sociological perspective by Matthias Wienroth.

\section{Mapping in DeVelopmental Biology}

At the outset of the ATRIA collaboration, Rundle outlined to Robinson the history of the investigation of developmental sequences in developmental biology and how this related to ongoing research in MBERC.

In their broadest sense, maps can be used to provide a representation of "the relative positions, or the spatial relations or distribution, of (an object or its components)" [1]. In this sense, maps have been at the heart of developmental biology for over 200 years [2]. Most recently, this is evident in the use of genetic maps, which show the relative positions and distance between genes on chromosomes. Yet the precedent for mapping in developmental biology was set by 19th-century embryologists in German universities, who described the development of vertebrate embryos using drawings and text [3]. Of most note here was the work of the zoologist-biologist Ernst Haeckel. Haeckel pioneered the idea of using the sequence of developmental events during the growth of an organism as a way of trying to get to the mechanistic basis for evolution. His central idea was the biogenic law, which proposed that the development of an animal reflected, or recapitulated, its evolutionary history [4]. In other words, more "advanced" 

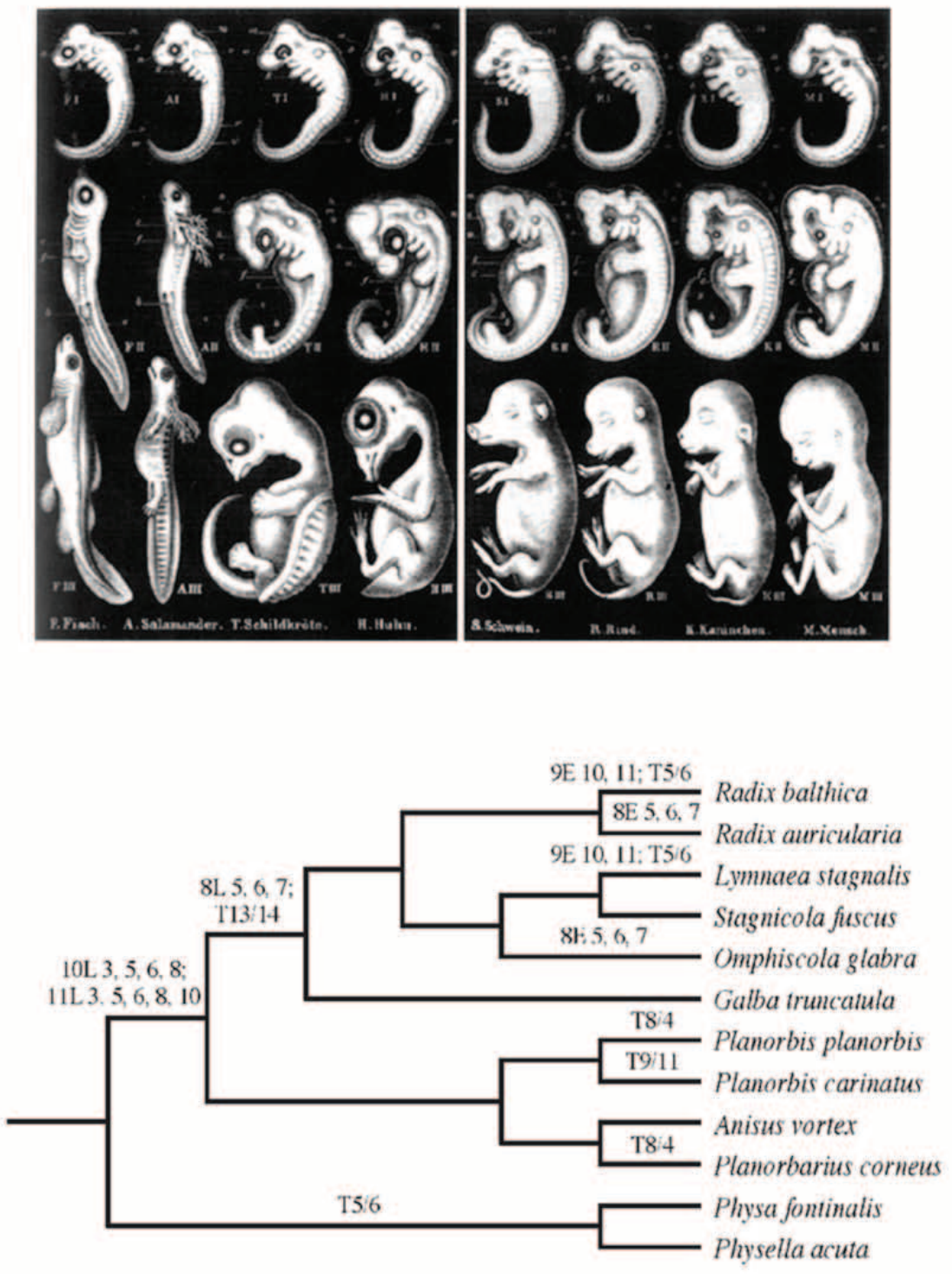

Fig. 1. Maps used in the study of developmental event sequences. (a) The drawings of developing vertebrate embryos used by Ernst Haeckel's illustrations in support of his Biogenic Law [4]; reproduced from Haeckel's original Anthropogeny, 1874. Development runs from top to bottom, and each column represents a species. (b) Plots of 14 developmental events on developmental timelines for 13 species of aquatic snail [6]. Development runs from left to right. The lines to the left of the timelines represent the species' phylogenies; species' relatedness is shown by connecting lines. Hence the two species at the start of the list are closely related. Some developmental events shift their relative timing between species, illustrating the phenomenon of heterochrony (see text). (๑) Smirthwaite, Rundle, Bininda-Emons and Spicer)

species such as mammals went through a "fish" stage during their development and their evolution progressed through the terminal addition of new features. Haeckel used detailed drawings of vertebrate embryos at various stages of development as key devices for conveying his ideas (Fig. 1a); these diagrams were arguably the first examples of mapping for the testing of a theory (i.e. the link between development and evolution) in developmental biology.

Haeckel's biogenic law is now generally regarded as flawed, but his approach of using developmental event sequences for investigating evolutionary relationships among species lives on in biology, and his iconic drawings are much reproduced. Moreover, another of Haeckel's concepts, heterochrony, where developmental events or stages show altered timing of occurrence between descendent species and their ancestors, and which Haeckel identified as a nuisance to his theory, is now viewed by many biologists as a key mechanism for species evolution [5].

One of the ways that contemporary scientists investigate the evolutionary importance of heterochrony is by first plot- ting the occurrence of different events on a timeline representing the development of a species (or an individual of that species) and then using computational techniques to compare the relative timing of developmental events (e.g. the formation of the heart) across species (Fig. 1b). Hence, the timing and sequence of developmental events are translated into data that are used to determine where evolutionary change has been accompanied by altered timing of developmental events. For Robinson, this contemporary plotting of developmental events onto developmental time lines and Haeckel's historical, iconic images of developing embryos arranged across developmental time involved a process of mapping (see Fig. 1a). These images also demonstrated a central role of the visual in developmental biological investigation, both in terms of the representation of the animals during development (e.g. Haeckel's drawings) and in the way that the data from these images were presented for scientific interpretation (contemporary mapping of developmental sequences).

\section{a Central Role of TECHNOLOGY IN MAPPING DEVELOPMENT}

Contemporary research on heterochrony and development in general has tended to focus on vertebrates. Given the difficulty of observing vertebrate development in vivo, however, most investigations that have aimed to compare developmental sequences across species have used information gathered by examining dead embryos of different ages. Biologists in MBERC have extended the use of the comparative approach but have focused on the embryos of invertebrates [6], many of which are transparent and, hence, can be observed in vivo.

Given the opportunities for scientific inquiry opened up by the use of these transparent embryos, there was a need to find a technology to automate this observation process. Consequently, a bio-imaging system for producing realtime, high-resolution videos of developing embryos was developed by Rundle's doctoral student Oliver Tills. Using a shutterless camera with a macro lens and bespoke lighting and stage mounting, this new system produced videos that tracked development from a single cell through to a fully formed snail. The increased temporal and spatial (depth of field) resolution provided by this new technology enabled the observation of physiological functions as well as fine- 
scale variation between embryos that had previously been undetectable.

Initially, Robinson saw the highly aesthetic nature of these high-quality videos as an interesting problem-they were, in a sense, complete in themselves. She therefore focused on the technical processes surrounding the production of the images and, again, the link with mapping data. In this case, numerical data were extracted from developing embryos both by human visual observation and through the use of image analysis programs, which measured frame-by-frame changes in movement of the embryo that were then plotted to show general movement patterns through time [7].

\section{Remapping: An Artistic Strategy To Manifest Data Dynamically in a Context Beyond Saience}

There was a second element to the scientific investigations on biological development being carried out in the MBERC that intrigued Robinson and would also play an important role in shaping ATRIA through a remapping process. Clearly, the videos of the embryonic development could be displayed as scientific data. They could be used to generate maps of developmental sequences, which would be of interest to evolutionary developmental biologists, or their movement could be analyzed and displayed for assessing questions in eco-physiology. But could these videos also be transposed into another context, extending their scientific meaning to a wider audience? Would a different context change shape and relational meaning of developmental mapping and its images by placing this biological information at the interface of two epistemic cultures-that of developmental biology and that of wider public understanding of embryonic development?

Context is key to perception and the reconstruction of meaning in art and in science. In the case of the ongoing work in MBERC, video and other data derived from embryos enabled the production of maps (scientific figures) that are vital for building on knowledge within a trajectory originating from Haeckel's work (see Fig. 1a). Haeckel's drawings of the developmental biology of vertebrates are still used widely within science, and outside of it, for their visual power. Yet Haeckel's biogenic law, which was underpinned by these drawings, is no longer seen as valid, and the power of the drawings has changed from theoretically ex- planatory to visually archetypical. As an object of scientific enquiry, they have experienced a shift in meaning while retaining their shape and relational power of visual stimulation due to their simplicity. Videos produced by MBERC scientists are three-dimensional and dynamic and hence much more "data-rich" than Haeckel's two-dimensional drawings. Data extracted from these videos are used to produce scientific diagrams to address complex issues such as animal development, allowing empirical observation to be shared (with other scientists) beyond the original specific observation and experiment. These diagrams also permit retracing data from the map to the stages between map-building and the initial observation-what the philosopher of science Bruno Latour has described as a process of "circulating reference" [8]. The ongoing interplay of data abstraction and enrichment in scientific practice between the initial, rich "matters of concern" and the reductionist yet ultimately more mobile "matters of fact" [9] is representative of daily practice in modern science-a cyclical process of "inscription" in which the "inscription device," i.e. any instrument used to provide "visual display of any sort in a scientific context," links to the production of "immutable or combinable mobiles" [10].

Circulation of such objects within the confines of a scientific community is viewed by Latour as integral to an increasingly bureaucratically driven set of practices whereby understanding of specific science is limited to that epistemic community. Such mobiles consist of assemblages of knowledge, the different contributors to, and users of, this knowledge and the context in which they are found. They incorporate a dual immutability - that of shape and that of relational meaning. Arguably, the meaning is the more significant here. Those scientific "immutable mobiles" may, however, become subject to challenge and unravel, becoming "mutable" [11]. Haeckel's drawings, for example, have undergone change pertaining to their relational meaning, if not their shape and role as archetypical illustrations.

Within the context of ATRIA, we can understand maps of developmental sequences or of cardiac function as artifacts, or objects, in a network of relationships that constitute developmental biology, its findings and emergent applications in, for example, healthcare, technology and popular culture, which reach out beyond the epistemic boundaries of science. For an object such as the map to be moved outside of developmental science and remain comprehensible begs the question of mobility and im/mutability. With the restaging of the data output within a different context-in a new site, for a diverse audience, with a critical focus on the observer in scientific knowledge production-the data's meaning could very well be reframed. Robinson began to envisage that such an alternative mapping process could be central to her work. Data pathways produced using imaging technology could be retraced and translated into a different medium, changing their physical shape. She envisaged this process as an experiment running in parallel to the science but with an outcome based on the presentation of information and data in a different medium and in a new context. This alternative map-the artwork-would incorporate sensory experience and exist in three- (as opposed to two-) dimensional space. There would be an emphasis on the role of the senses, immersion and the body as a means of response. It was intended that this new map would be introduced-and be meaningful - to wider audiences beyond the scientific community.

The artistic strategy chosen retraced data pathways using what we term "sound" in order to form an immersive, sensory 3D map with the intention of reintroducing the body into scientific epistemology, which negates the corporeal presence of the observer. This artwork, through re-presenting data as sound/image at liminal and vibrational levels, attempted to blur the distinction between observer and observed, rendering reception of data experiential in the extreme. The approach adopted was chosen to contrast with scientific mapping, which tends to condense and depict phenomena on a 2D surface. In this attempt at changing the shape of the object "map"-toward an emergent 3D artwork-the relational meaning had to remain immutable in order to convey the scientific interpretation of the immersive sensory experience of the audience; the context of a map was retained as a walkable, auditory experience.

In the making of the artwork, sound artist David Strang was involved in conversations with the team about the possibilities of transposing data from the videos depicting embryonic gestation as sound. Robinson wanted to extend experimentation carried out in previous projects where she had developed strategies that would reveal un-thought or "unconscious" structures, such as the 


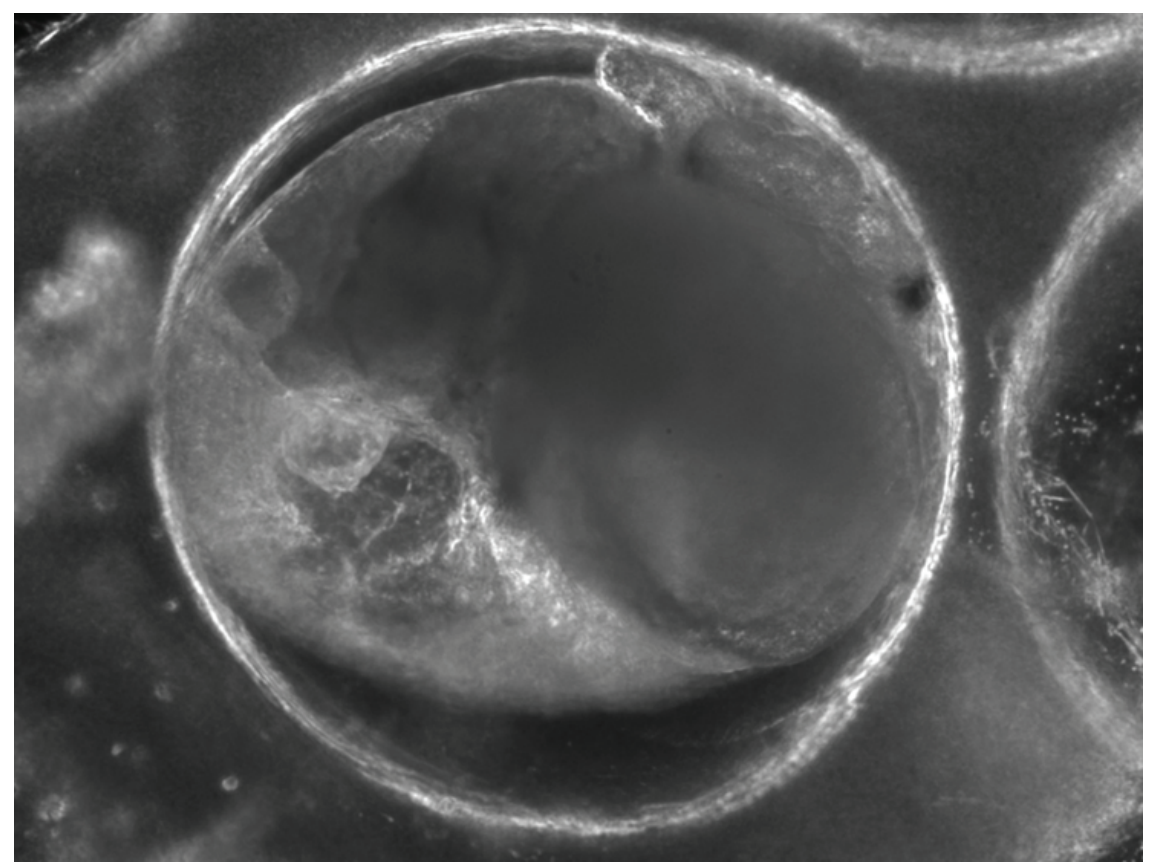

Fig. 2. An encapsulated embryo of the marine snail Littorina obtusata. This still image is taken from one of the videos used to create ATRIA. The two-chambered true heart can be seen through the transparent shell on the left of the animal. To the right of the animal the eye can be seen and to the left of this the oval shape of the foot. The egg capsule is approximately $0.5 \mathrm{~mm}$ in width. (Photo $@$ Oliver Tills)

principles of the mind/body division, and its inherent reductionism, that underlie the construction of knowledge in science. Highly aware of the microscopic, "vulnerable" nature of the encapsulated embryos, she aimed to transpose information generated by the observed organism into a dynamic form that was less contained and remote than its form as a standard scientific output. Historian of media art and science studies scholar Douglas Kahn observes to that effect that

sounds can be heard coming from outside and behind the range of peripheral vision, and a sound of adequate intensity can be felt on and within the body as a whole, thereby dislocating the frontal and conceptual associations of vision with an all-around corporeality and spatiality [12].

As an artist, Robinson was interested in the exploration of unfamiliar viewpoints and the subsequent effects on perception. Through using sound, she wanted to explore the displacement of scientific material and also of the viewer [13]. Sound, in contrast with the more distancing mechanism of sight, would permeate the space of the viewer. To this end, lowfrequency sound-something more like vibration-became increasingly important, as this meant that the artwork would be indivisible from the viewer, overturning the observer/observed relationship.

\section{REMAPPING HEART DEVELOPMENT}

The final strands in developing the ATRIA installation involved the choice of which aspect of developmental biology and, hence, which videos to use as a focus and where to site the work. The choice of video stemmed from an upgrading of the bio-imaging system. MBERC doctoral student Oliver Tills added a new camera lens to his set-up around six months into Robinson's residency. This lens allowed the production of embryo videos with an even greater depth of field, giving a three-dimensional view of structures such as the developing heart. This technological advance led to the detection of "additional" developmental features previously unseen within the laboratory. For example, small twitches of the heart before it began beating continuously were now identified. This opened up new research possibilities that could use video footage to monitor the development of the cardiovascular system in aquatic invertebrates.

One project that utilized this newfound technology seemed to offer the most possibilities: an investigation of how the development of the cardiovascular system in the flat periwinkle (Littorina obtusata) (a common inhabitant of intertidal rocky shores in the U.K.) responded to low oxygen levels [14]. Like many molluscs, this species of snail has during its development a complex circulatory system, in this case two hearts: a transitory, larval heart and a "true" heart (Fig. 2). The larval heart begins beating first, a few days into development; a few days later the true heart begins beating and then, for several days, the two hearts beat at the same time; finally, the larval heart is absorbed and stops beating, and the true heart remains and takes over as the main driver of circulation in the juvenile and adult snail (Fig. 3). Videos of both the larval and adult heart were used as the basis for ATRIA.

The extraction of data from these videos and the use of these data to create sound were performed using the program Max/MSP [15] and, in particular, the computer vision (CV) library cv.jit created by Jean-Marc Pelletier [16]. The external object $c v$.jit.opticalflow was used to produce an output based on the velocity of the pixel movement to allow a focus on the movement of the heart as it was beating. Any unwanted noise was then removed from these images using $c v$.jit.framesub. The resulting image at this stage was a collection of pixels showing only the movement of the heart. This

Fig. 3. Development of heart function in the marine snail Littorina obtusata during early development. This graph shows the change in the beating of the two (larval and true) hearts across the first $\mathbf{2 2}$ days of development (larval heart $=$ dashed line). There are three main phases: (1) beating of the larval heart only (days 0-11); (2) both larval and true hearts beating (days 11-16); (3) only the true heart beats (day 22 on) - at this stage the larval heart typically stops beating or beats only sporadically. Reproduced from Bitterli et al. [14] with permission; doi: 10.1242/jeb.067967, <jeb.biologists.org>. (@ Bitterli, Rundle and Spicer)

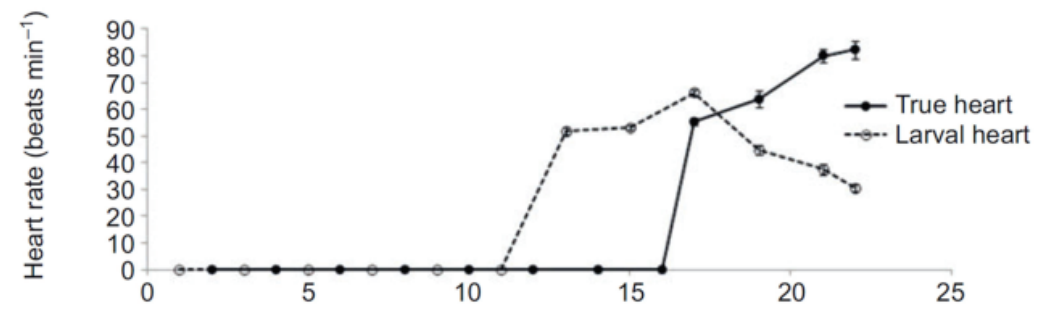




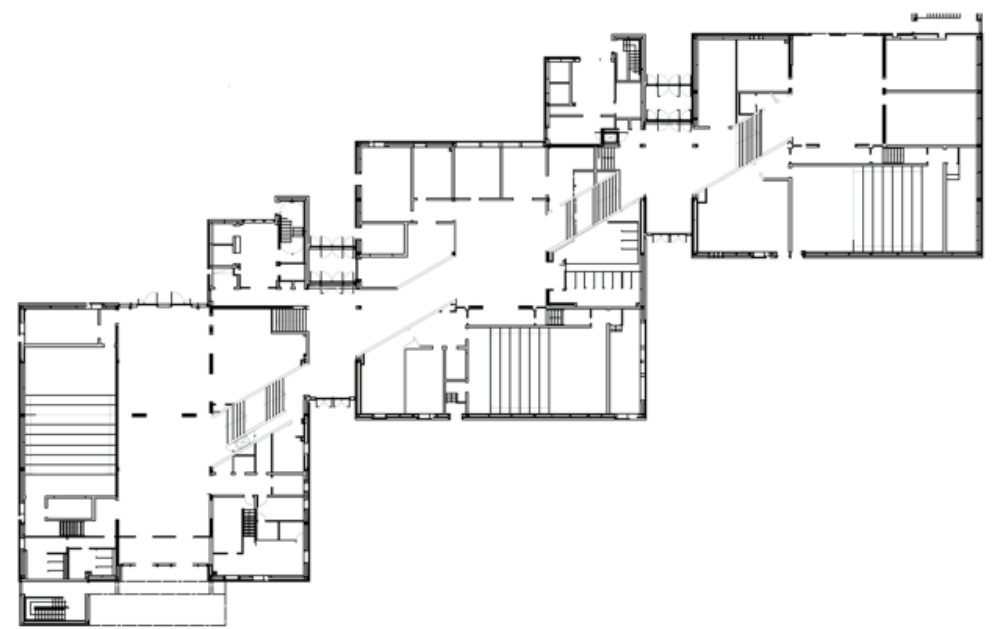

Fig. 4. Plan of the ground floor of the Portland Square Building. This is a 5-story building, and the three atria into which sound was projected can be seen at the center of each of the three blocks. The atria run from the ground floor to ceiling, and the sequence used in the text, from first to third, runs from left to right. (๔) Plymouth University [Estates])

Fig. 5. Atrium 2 in the Portland Square Building. Three of the speakers used to generate sound are visible, as are the stairs leading through to Atrium 3. (ATRIA (C) Robinson, Strang and Rundle. Photo @ Deborah Robinson.)

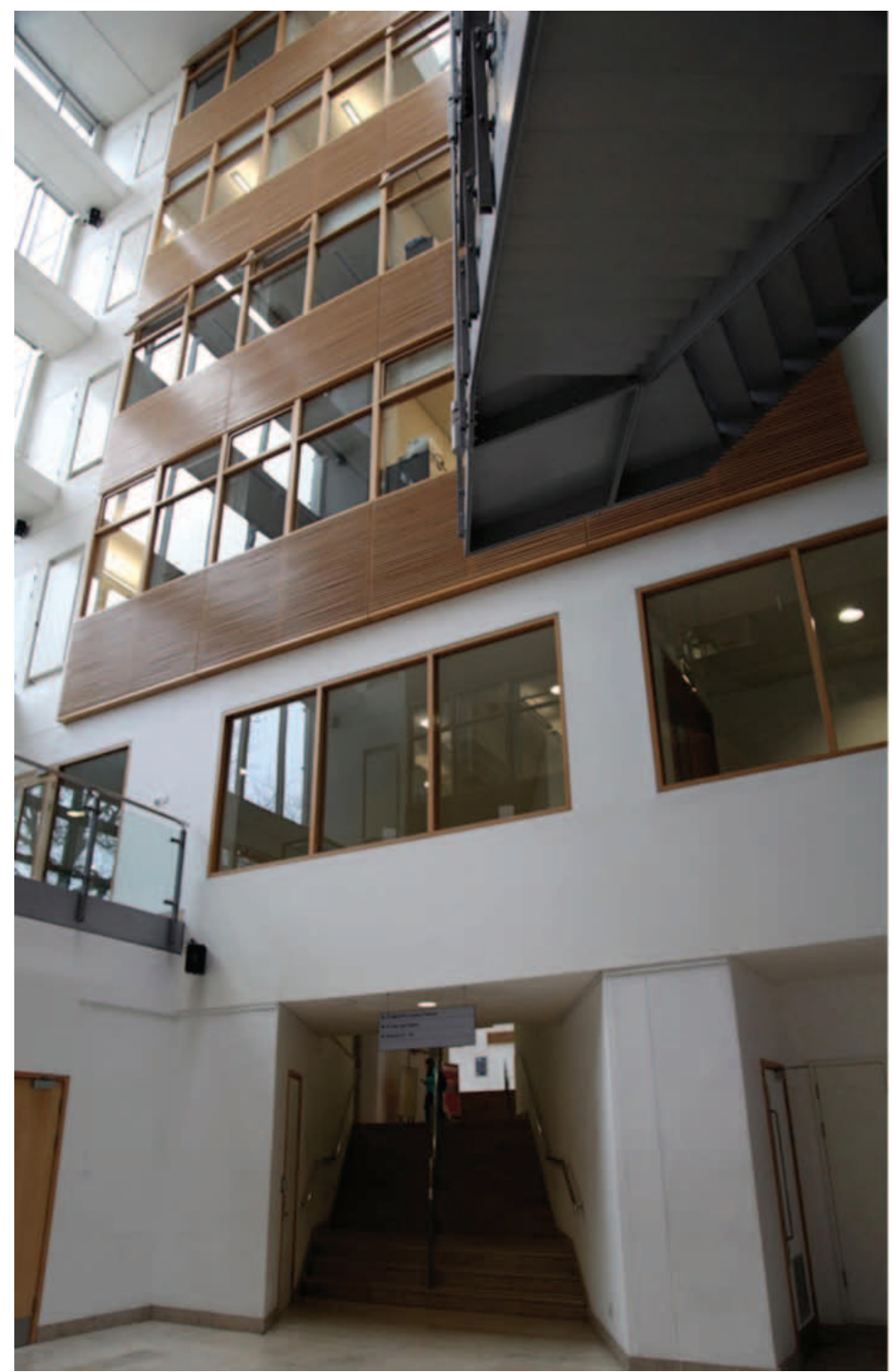

collection of pixels was then analyzed according to the mass of active pixels on the screen using $c v$.jit.mass so that, as the heart pumped, it generated a flow of numbers rising and falling "in synch." This number stream was then used to pulse a low-frequency oscillator and produce the sonic output for the speakers. Two different pitches were chosen for the two different stages of the development of the embryo. The larval heart was pitched slightly higher than the true heart in order for the difference to be clearly audible (not just from a difference in rhythm) when the sounds from the two hearts overlapped.

The next stage in the development of ATRIA was the decision on where to site it. The Portland Square Building on the main campus of Plymouth University was chosen. This building comprises three equally sized blocks, each of which contains an atrium (Figs 4 and 5). It also contains a 56 -speaker system that can be used to play sound into each of the atria separately. The flexible nature of Max/ MSP meant it was possible to connect with this large configuration of speakers. This offered the exciting opportunity to project sound generated from the three different stages of the development of the circulatory system in Littorina obtusata (1: larval heart only, beating; 2: larval heart and true heart beating together; 3: true heart only, beating) into the three different atria. A playback sequence was set up, in which the larval heart began to play through a set of speakers in the first atrium of the Portland Square building. After three minutes this sound then started in the middle atrium. At this stage the sound generated from the adult heart video was gradually faded in to the middle atrium, and the two heart rhythms then played together in this atrium for three minutes. These two beats were then both faded out in the second (middle) atrium as the adult heart was faded on in the third atrium. Hence the sequence ended with just the true/ adult heart playing at the opposite end of the building from where the sequence began. This sequence, which had a duration of approximately eight minutes, was then repeated as a continuous loop.

The ATRIA installation was presented on the evening of 15 March 2011. First, an introductory talk was given in which the scientific and technological background to the artwork as well as the work itself were presented (Fig. 6). The video on which the work was based was also shown, providing the audience, a mixture of scientists and artists, with an experience of the source of the sound data. 

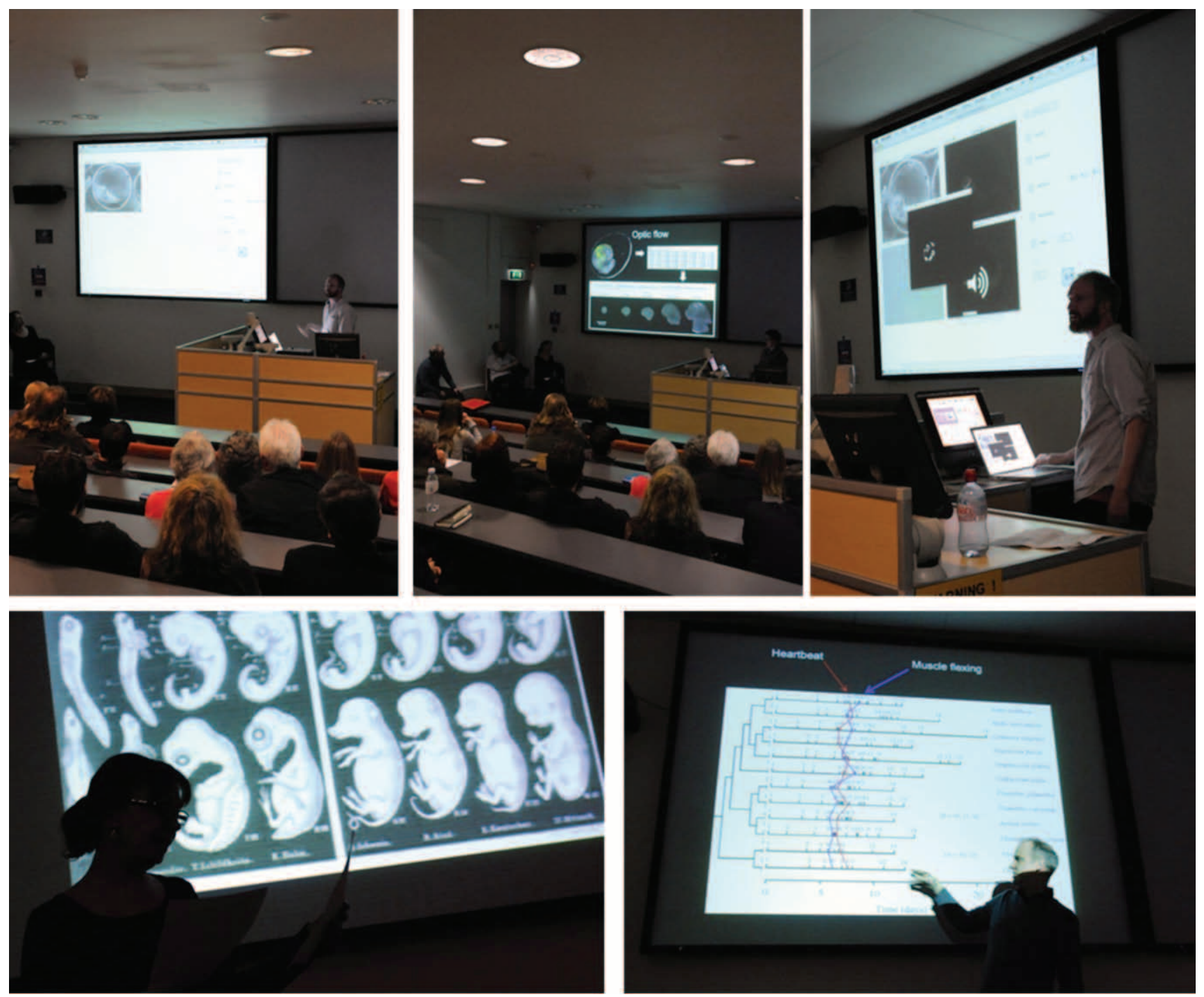

Fig. 6. Presenting the ATRIA project. (๑ Robinson, Strang and Rundle. Photos @ Plymouth University [Media Services])

The audience then entered the building space silently but was not given any directions on how to engage with the work.

The installation conveyed the developing snail's cardiovascular development-reflecting established scientific knowledge produced at MBERC-in an affective experimental setting for the observer. The interpretations of the audience members, who experienced the auditory and vibrational transposition of experimental data, were influenced by the introductory talk. This explanatory aspect of the presentation was deliberately separated from the embodied experience of engaging with the immersive and resonating sound heightened through low levels of light. Some audience members chose to walk "in order" either from Atrium 1 through to Atrium 3 or vice versa; others stood predominantly in one atrium and "sampled" the other two sporadically. From the point of view of the MBERC scientists involved, an element of their research (the data derived from human observation of videos of cardiovascular development) had been transposed into the installation. Yet this remapping of ATRIA did not constitute an immutable mobile. Instead, it might rather be described as a "mutable mobile," constituted in the fluidity of its relations [17] and the multiplicity of the practices in which it is enrolled [18]. The remapping, and restaging, of the developmental map drew on scientific practice and knowledge in developing artistic experience. Revising the notion of the map and its content into an immersive sensory three-dimensional experience presented the fluidity of the notion of a map but also emphasized the artistic practice by drawing attention back to the body of the observer. Indeed, in line with Latour's ideas [19], we can suggest that the biological "matters of fact" were retraced in the remapping process into their "matters of concern"-the rich assemblage of individuals and their experiences and ideas leading toward observations; the observable and not yet data-ized things of the snail, its hearts and its embryonic development; and the scientific context in which such knowledge production takes place-and in which the body of the observer is so often ignored.

\section{Concluding Remarks}

In ATRIA, cognitive data about cardiovascular development were remapped into a sensual context, retaining biological meaning yet changing the shape of the map and its experience in a fluid reimagining of the practices of knowledge production and "sensing." The scientific knowledge remained unchanged, or immutable, yet mobile, as the developmental stages of larval and "real" hearts provided the basis for the artistic installation. The shape became mutable in this challenge to the blanking out of the 
individual observer, who has so much influence on the process of knowledge production [20].

In this paper, we have outlined how the ATRIA project was developed through our collaboration associated with Robinson's time as artist in residence within MBERC, describing the scientific underpinning to the work and the dynamic way in which discussions, technology development and philosophical ideas led to the presentation of a sound installation. We hope that we have shown how critical scholarly engagement with scientific knowledge can contribute to artistic gestation at the art-science interface and hope that this description sheds light on the often blurry connections and interactions between scientific and artistic practice.

\section{Acknowledgments}

We are very grateful to Oliver Tills for his contribution to scientific discussion and for providing the video footage that was so integral to ATRIA. Thanks also to John Spicer and Tabitha Bitterli for their input to art-science discussions. Funding for DR's residency was very generously provided by the Faculty of Science and Technology at Plymouth University.

\section{References}

Unedited references as provided by the authors.

1. Oxford English Dictionary.

2. Hopwood, N. (2007) A history of normal plates, tables and stages in vertebrate embryology. International Journal of Developmental Biology 51: 1-26.

3. Rundle, S.D. (2012) Disrupting the ideal: Variation in embryos as a model for Transpositions. In: Transpositions, exhibition catalogue, University of Plymouth Press.

4. Richardson, M.K. \& Keuck, G. (2002) Haeckel's
ABC of evolution and development. Biological Reviews 77: 495-528.

5. Gould, S.J. (1977) Ontogeny and Phylogeny. Harvard University Press, Cambridge, Massachussetts, U.S.

6. Smirthwaite J.J., Rundle S.D., Bininda-Emonds O.R.P. \& Spicer J.I. (2007) An integrative approach identifies sequence heterochronies in pulomonate snails. Evolution and Development 9: 122-130.

7. Tills, O., Culverhouse, P., Spicer, J.I. \& Rundle, S.D. (2013) An automated approach for identifying embryonic developmental stages. BMC Bioinformatics 14:37.

8. Latour, B. (1999) Circulating Reference. Sampling the Soil in the Amazon Forest, in: Pandora's Hope. Essays on the Reality of Science Studies, Cambridge, MA, and London: Harvard University Press: 24-79.

9. Carroll, P. (2012) Water and technoscientific state formation in California, Social Studies of Science 42(4): 483-488.

10. Latour, B. (1987) Science in Action, Cambridge, MA: Harvard University Press: pp. 68 and 226-227.

11. M'charek, A. (2000) Technologies of population: Forensic DNA testing practices and the making of differences and similarities, Configurations 8(1): 121-158.

12. Kahn, D. (1999) Noise, Water, Meat: A history of sound in the arts, MIT Press: p. 27.

13. McCluhan, M., Fiore, Q. \& Agel, J. (1996) The medium is the massage: An inventory of effects. HardWired, San Francisco.

14. Bitterli, T.S., Rundle, S.D. \& Spicer, J.I. (2012) Development of cardiovascular function in the marine gastropod Littorina obtusata (Linnaeus). Journal of Experimental Biology 215: 2327-2333.

15. <http://cycling74.com/>

16. <http://jmpelletier.com/cvjit/>

17. De Laet, M. \& Mol, A. (2000) The Zimbabwe bush pump. Mechanics of a fluid technology, Social Studies of Science 30 (2): 225-263.

18. Mol, A. (2002) The Body Multiple. Ontology in Medical Practice, Durham, NC: Duke University Press.

19. Latour, B. (2004) Why has critique run out of steam? From matters of fact to matters of concern, Critical Inquiry 30(2): 225-248.
20. Latour, B. \& Woolgar, S. (1986) Laboratory Life. The Construction of Scientific Facts, Princeton, NJ: Princeton University Press.

Manuscript received 8 November 2012

Simon Rundle is Reader in Aquatic Biology and coordinator of the Marine Biology and Ecology Research Centre at Plymouth University. His research focuses on links between development, ecology and evolution, but he has recently developed an interest in interdisciplinary research, in particular parallels between artistic and scientific practice.

Deborah Robinson is an artist whose work explores methodological contrast between the disciplinary boundaries of art and science using this to generate work that raises questions involving agency, transposition, context and meaning. She is Associate Professor (Reader) in Contemporary Art and co-ordinator of the $A R C$ research group at Plymouth University. Her work has been exhibited in the U.K., Germany, and the U.S.A.

David Strang is an artist who works with sound and interactive elements. His work looks closely at the natural surroundings we live in and amplifies certain aspects to heighten our perception of space/place. Recent work includes site-specific installation, fieldrecording, networks, re-appropriating media objects, hacking and noise. He has collaborated and exhibited with visual artists and scientists as well as exhibited solo work in the U.K., Europe, Russia and the U.S.A.

Matthias Wienroth is Research Fellow at the Northumbria University Centre for Forensic Science. As a science and technologies scholar, he is particularly interested in understanding the ways that knowledge and meaning are produced in interactions of very different perspectives, e.g. in art-science, science policy, and technoscience. 


\section{Thanks to Our Reviewers}

The Leonardo publications are peer reviewed: before acceptance, every article is reviewed and commented on, usually by three anonymous reviewers. Based on these reviews we make a decision whether to publish the text.

Leonardo covers a wide variety of fields, many of them interdisciplinary and often in emerging areas of experimentation, research and scholarship. Our peer reviewers help us focus on new topics of interest, as other areas may move out of the center of experimentation and become part of established disciplines. In very new areas of inquiry it is often difficult for us to identify three reviewers competent to review the work; in these cases we may ask the authors themselves to suggest new reviewers for our peer review panel. The Leonardo Network in a very real sense grows and evolves through this process of collaborative filtering.

We wish to take this opportunity to thank some of the reviewers who in the past year have on a volunteer basis spent time writing very thoughtful reviews that both help us make a publication decision and help the authors to improve their texts:

Liliana Albertazzi, Christine Assaiante, Marc Battier, Sinan Bokesoy, Claus-Christian Carbon, David Carrier, Michael Corballis, Mellisa Coleman, Evelina Domnitch, Frank Dufour, Michael Early, David Em, Michele Emmer, Jayanne English, James Enns, Kathryn Evans, Sidney Fels, Jessica Field, Ken Fields, Michael Fowler, Jean Gagnon, Scot Gresham-Lancaster, Amanda Gluibizzi, Brian Harnetty, Amy Ione, Susan Iverson, Janis Jefferies, Mauri Kaipainen, Jeremy Kargon, Kim Knight, Francois-Joseph Lapointe, Patrice Le Gal, Guillermo Lemarchand, Jerrold Levinson, Patrick Lichty, John Mallinckrodt, Aleksandra Manczak, Edgar Meyer, Rosemary Mountain, Frieder Nake, Michael Neff, Stephan Neuhauss, Michael O'Shea, Jin Wan Park, Sidney Perkowitz, Lucy Petrovic, Cliff Pickover, Sheila Pinkel, Patricia Pisters, Vincent Racaniello, Keith Rayner, Joana Ricou, Robert Root-Bernstein, Mark Rosen, Susan Ryan, Sundar Sarukkai, Aparna Sharma, Tami Spector, John Stein, Anne Swartz, Tibor Tarnai, Robert Thill, Yu-Chuan Tseng, Kim Veltman, Julian Voss-Andreae, James Faure Walker, Stephan Wensveen, Louise Whiteley, Xin-She Yang, Kang Zhang, Jonathan Zilberg 